\title{
Intranasal Oxytocin Administration Ameliorates DSS-induced Abnormal Stress-related Behavior and Intestinal Inflammation in an IBD Mouse Model
}

\author{
Chuanyong Liu ${ }^{1}$, Jinghui Liang ${ }^{2}$, Haixia $\mathrm{Li}^{3}$, Jianchun $\mathrm{Zhu}^{3}$, Dandan $\mathrm{Dou}^{3}$, Yang $\mathrm{Li}^{1}$, and \\ Jingxin $\mathrm{Li}^{3}$ \\ ${ }^{1}$ Shandong University \\ ${ }^{2}$ Shandong University Qilu Hospital \\ ${ }^{3}$ Physiology
}

October 2, 2020

\begin{abstract}
Inflammatory bowel disease (IBD) comprises Crohn's disease and ulcerative colitis. In patients with IBD, intestinal inflammation and psychological comorbidities affect the quality of life. Evidence for the effectiveness of antipsychotics drugs or psychological therapies in patients with IBD is currently lacking. However, several studies have reported that intranasal oxytocin (OT) administration is effective in individuals with psychological disorders. Therefore, in this study, we evaluated the effects of intranasal OT on psychological disorders, using an IBD mouse model established via dextran sodium sulfate (DSS). Our results showed that intranasal OT improved DSS- induced abnormal stress-related behavior and restored the DSS-induced alterations in nNOS/NO, oxytocin receptor (OTR), pERK/ERK and BDNF expression in the hippocampus. Intranasal OT also ameliorated intestinal inflammation. The activity of the hypothalamic-pituitary-adrenal axis and the sympathetic-adrenal medulla axis were also altered by intranasal OT, without affecting the peripherally-secreted OT. Thus, while intranasal OT administration increased the concentration of OT in the hypothalamus compared to that in the untreated IBD mouse, the OT levels in the serum did not change. Intranasal OT increased the percentages of the M1 and M2 type macrophages and regulatory $\mathrm{T}$ (Treg) cells in the IBD mice, in contrast it decreased the M1/M2 ratio and the percentage of NKp46+NK cells in the spleen. We found that the protective effects of intranasal OT administration on impaired stress-coping behavior and intestinal inflammation could be abolished by splenectomy. In conclusion, the present study demonstrates that intranasal OT can ameliorate DSS-induced abnormal stress-related behavior and intestinal inflammation.
\end{abstract}

\section{Introduction}

Inflammatory bowel disease (IBD) represents a group of inflammatory conditions of the small intestine and colon, including Crohn's disease (CD) and ulcerative colitis (UC). IBD is considered a primary health issue, with increasing prevalence and incidence ${ }^{1}$. However, the disease processes associated with IBD are not limited to the gastrointestinal tract. These disease processes may spread to the brain, thus increasing the risk of psychological disorders through changes to the hypothalamus-pituitary-adrenal (HPA) axis, inflammatory cytokines, and neurotransmitters ${ }^{2-5}$. For instance, depression is closely related to a clinical relapse of IBD; the occurrence of depression appears to shorten the time to clinical relapse, and increase both the onset and complications of the relapse ${ }^{6-8}$. The presence of a psychological disorder can also adversely affects the course of IBD, which can then, in turn, exacerbate psychological wellbeing ${ }^{9}$. Moreover, while psychological therapies may not have any beneficial effects on IBD activity, antidepressant and anti-anxiety drugs can reduce the effects of anti-inflammatory treatments. Further, they may even aggravate the symptoms and 
cause harmful side effects ${ }^{1011}$. Therefore, there is an urgent clinical need for the development of improved treatments for IBD.

Oxytocin (OT) is a neuropeptide produced by the hypothalamus and paraventricular nucleus and is released by the posterior pituitary gland ${ }^{12}$. OT plays an important role in both nonsocial and social functions, including reproduction, pain perception, immune regulation, maternal behavior, sexual behavior, pair bonding, aggression, social memory and stress ${ }^{13-15}$. Recently, OT and its receptor have been shown to have a substantial effect on several psychiatric disorders, including anxiety and autism spectrum disorder ${ }^{1617}$. The effects of OT on the brain have been previously investigated via intranasal administration of OT. Intranasal administration bypasses the blood-brain barrier allowing the delivery of OT to specific brain regions ${ }^{18-21}$. Moreover, because OT is absorbed and metabolized in the intestine and has a short half-life in blood, the oral administration of OT is problematic ${ }^{22}$. In addition, intranasal administration is convenient and noninvasive.

Interestingly, Reichmann previously reported that dextran sodium sulfate(DSS)-induced colitis impaired normal behavior in mice during a water avoidance stress test (WAST). Thus, DSS-treated mice exhibited an abnormal stress-coping ability and presented a depression-like phenotype ${ }^{23}$. The implications of intranasal OT treatment on the regulation of IBD-induced psychological disorders remains to be determined. Here, to provide insights into this phenomenon, we investigated whether intranasal OT administration can improve abnormal stress-related behavior in mice with colitis.

\section{Materials and Methods}

\section{Animals}

The animals used in this experiment were in line with the "Guidelines for the Care and Use of Laboratory Animals" formulated by Shandong Province, China, and approved by Medical Ethics Committee for Experimental Animals, Shandong University School of Basic Medicine Sciences (ECAESDUSM 2014056). We separated wild-type C57BL6/J mice (male, 6-8 weeks of age) randomly. The investigators were blinded to the group allocation. Animals were euthanized by cervical dislocation after DSS administration.

\section{Intranasal administration}

Atosiban is a competitive OT receptor antagonist ${ }^{24}$. Both OT and atosiban have been shown to be bioavailable when administered intranasally ${ }^{25,26}$. The intranasal dose of normal saline was $20 \mu \mathrm{l} /$ day. The intranasal dose of OT was $1 \mathrm{mg} / \mathrm{kg} /$ day, diluted in $20 \mu \mathrm{l}$ saline. The intranasal dose of atosiban was $10 \mathrm{mg} / \mathrm{kg} / \mathrm{day}$, diluted in $20 \mu \mathrm{l}$ saline. Mice were handled daily one week before the start of intranasal treatments to reduce the stress associated with the procedure. Two days before DSS-induced colitis, mice had been received an intranasal saline, OT or OT with atosiban. Mice were picked up by the scruff of the neck and exposed in a supine position to immobilize them for the intranasal administration. This position of head was maintained throughout procedure preventing drainage of drug solution to trachea and esophagus. The total volume of $20 \mu$ l solutions was administered by pipette in $5 \mu$ l drops in alternating naris every 30 seconds. The drop was placed at the naris opening while occluding the opposite naris allowing the animal to snort the drop into the nasal cavity.

Study design

Behavior and biochemical test comprised four groups: NC, DSS, DSS+IN OT, DSS+IN OT+IN atosiban. NC group was treated with plain drinking water for seven days; untreated DSS group was treated with $2 \%$ DSS powder in plain drinking water for 7days; DSS+IN OT group was treated with $2 \%$ DSS powder in plain drinking water and intranasal administration of OT for seven days. DSS+IN OT+IN atosiban group was treated with $2 \%$ DSS powder, intranasal administration of OT and atosiban forseven days. At seventh day, half of each group were submitted to a water avoidance stress test for 10 minutes. The other half were sacrificed to perform a biochemical test.

In the second series of experiments, chemical colitis was induced in mice 15 days after splenectomy. Splenectomy mice were assigned to the following experimental groups: splenectomy, DSS + splenectomy and DSS+IN 
OT + splenectomy. With NC, DSS and DSS+IN OT groups together, these three groups were performed the same experiments as the first series. The design can be seen in Supplementary figure.

\section{Water avoidance stress test (WAST)}

We performed water avoidance stress test as previously described ${ }^{23}$. The equipment of water avoidance stress was used to provide an assessment of stress response. After 7-day drug treatment, each group of mice in behavioral test were placed on a small platform in the center of water-filled tank, the level of the water $\left(25{ }^{\circ} \mathrm{C}\right)$ in the tank being $1 \mathrm{~cm}$ below the platform. The stress procedure was carried out in a brightly lit room. Immediately after submission to WAS, the behavior of mice was recorded for 10 min to evaluate their behavioral reaction to stress and recorded. Time being moving, time being immobile and time spent on grooming on the platform were measured. After exposure to WAST, the mice were returned to their cage.

\section{Evaluation of colitis}

The severity of colitis was evaluated by continuously weight change, colon length, disease activity score, macroscopic damage score, histological score and colonic cytokines levels following an established protocol 27.

For assessing disease activity score, two investigators scored the results by body weight loss, stool consistency and rectal bleeding in a double-blinded manner. The scores were quantified as follows: body weight loss on a scale of $0-5$ for $<5 \%, 5-10 \%, 10-15 \%, 15-20 \%, 20-25 \%,>25 \%$, respectively; stool consistency on a scale of 0-2 for normal, soft, liquid, respectively; and rectal bleeding on a scale of $0-1$ for absent, present, respectively.

For assessing macroscopic damage score, two investigators scored the results by presence of strictures and hypertrophic zones, adhesion areas and intraluminal hemorrhage in a double-blinded manner. The scores were quantified as follows: presence of strictures and hypertrophic zones on a scale of 0-3 for absent, one stricture, two strictures, more than two strictures, respectively; mucus on a scale of $0-1$ for absent, present, respectively; adhesion areas between the colon and other intra-abdominal organs on a scale of $0-3$ for absent, one adhesion area, two adhesion areas, more than two adhesion areas, respectively; intraluminal hemorrhage on a scale of $0-1$ for absent, present, respectively; erythema on a scale of $0-2$ for absent, presence of a crimsoned area $<1 \mathrm{~cm}^{2}$, presence of a crimsoned area $>1 \mathrm{~cm}^{2}$, respectively; and ulcerations and necrotic areas on a scale of $0-2$ for absent presence of a necrotic area $<1 \mathrm{~cm}^{2}$, presence of a necrotic area $>1 \mathrm{~cm}^{2}$, respectively.

For assessing colonic histology score, two investigators scored the results by epithelial surface damage, the loss of crypts and inflammatory infiltration in a double-blinded manner. The scores were quantified as follows: crypt damage on a scale of 0-4 for no damage, basal one-third damaged, basal two-thirds damaged, only surface epithelium intact, and entire crypt epithelium lost, respectively; depth of injury on a scale of $0-3$ for no, mucosal, mucosal and submucosal, and transmural injury, respectively; and inflammatory infiltration on a scale of $0-3$ for no, slight, moderate, and severe inflammation, respectively.

\section{Western Blot Analysis}

Hippocampus samples were collected in RIPA buffer (Beyotime, Shanghai, China) and centrifuged for 20 min at $12000 \times \mathrm{g}$ and $4^{\circ} \mathrm{C}$. Then we collected supernatants calculated protein concentration. We took 40 to $60 \mu \mathrm{g}$ protein per sample, and performed protein separation by SDS-PAGE electrophoresis. After blocked by the $5 \%$ milk powder at room temperature for $2 \mathrm{~h}$, the membranes were incubated with primary antibodies against pERK (Cell Signaling Technology, \#4370), ERK (Cell Signaling Technology, \#4695), OTR(Abcam, \#ab217212), nNOS (Cell Signaling Technology, \#4231), GAPDH (Cell Signaling Technology, \#5174) at $4^{\circ} \mathrm{C}$ overnight. The membrane was incubated with a secondary antibody at room temperature for $1 \mathrm{~h}$. We detected signals by enhanced chemiluminescence following the manufacturer's recommendations.

\section{ELISA}

BDNF (CUSABIO, \#CSB-E04505m), Cortisol (CUSABIO, \# CSB-E05113m), ACTH (CUSABIO, \#CSBE06874m), CRH (CUSABIO, \#CSB-E14068m), catecholamine (Fitzgerald, \#3-CAT ELISA Kit), OT (CU- 
SABIO, \#CSB-E09245m), Ach(CUSABIO, \#CSB-E04744m), TNF- $\alpha($ CUSABIO, \#CSB-E04744m) and IL$1 \beta$ (CUSABIO, CSB-E08054m)was detected by using relevant ELISA kit following the manufacturer's instructions.

\section{Measurement of NO}

NO was measured by nitric oxide assay kit (Nanjing Jiancheng Bioengineering, \#A012) following the manufacturer's instructions.

\section{Splenectomy}

Splenectomy was performed 15 days before DSS-induced colitis. Mice were fasted for eight hours before surgery. Then mice were anesthetized by intraperitoneal injection of pentobarbital $50 \mathrm{mg} / \mathrm{kg}$. The spleen was removed after abdominal surgery and ligation of blood vessels. After the surgical intervention, mice were monitored daily to examine their health state. We reconfirmed the absence of spleen when the specimens were retained.

\section{Flow Cytometry Assays}

We followed previous research method to perform flow cytometry assay ${ }^{27}$. For isolation of splenocytes, spleen was mechanically dispersed through a 100um cell-strainer and washed with PBS. The cellular suspension was then centrifuged at $1500 \mathrm{r}$ for $10 \mathrm{~min}$ at $4^{\circ} \mathrm{C}$, then incubated with Red Blood Cell Lysis Buffer (Solarbio, \# R1010) for 5 min in the dark. Afterward, samples were centrifuged at $1500 \mathrm{RCF}$ for $10 \mathrm{~min}$ at $4^{\circ} \mathrm{C}$, then washed with PBS and re-suspended in $1 \mathrm{~mL}$ PBS. The obtained cellular suspension was stained with fluorescent antibodies.

Before immunofluorescent staining, the cellular suspension of splenocytes was incubated with Fc (Biolegend, \#101319) for $10 \mathrm{~min}$ in the dark at $4^{\circ} \mathrm{C}$, in order to block non-specific binding sites for antibodies. The following antibodies were used:CD11b (FITC, Biolegend, \# 101205), F4/80(Percpcy5.5, Biolegend, \#123127), CD206(PE, Biolegend,\#141705), CD86(APC, Biolegend,\#105011), CD4(FITC, Biolegend, \#100405), CD25(APC, Biolegend, \#102011), Foxp3(PE, Biolegend, \#126403), CD3(FITC, Biolegend, \#100203), NKp46(PE, Biolegend, \#137603).

Samples were analyzed using CytExpert software. The spleen cell populations were defined as follows: Treg cell $\left(\mathrm{CD} 4^{+} \mathrm{CD} 25^{+} \mathrm{FoxP}^{+}\right)$, M1 macrophage (CD86+F4/80+CD11b+), M2 macrophage $(\mathrm{CD} 206+\mathrm{F} 4 / 80+\mathrm{CD} 11 \mathrm{~b}+)$, NK cell (CD3-NKp46+). Percentages were reported to the total number of splenocytes or MLN cells of each mouse to calculate the number of cells per population.

\section{Statistical Analysis}

The visualized patterns of western blot were analyzed by ImageJ 1.48 and Image Lab 4.1.0 to transfer images to digits. All data, each one representing the outcome of a single animal, were presented as means \pm SEM. Comparisons among experimental groups were made using analysis of variance (one-way or two-way ANOVA) followed by the Tukey-Kramer multiple comparisons post-test when $P<0.05$, chosen as level of statistical significance, was achieved. All analyses were performed using Prism 4 software (GraphPad Software Inc., San Diego, CA, United States).

\section{Result}

\section{Intranasal OT improved the DSS-induced abnormal stress-related behavior of mice}

Analysis of the WAST results indicated that the IBD mice showed a decreased tendency to explore (Fig.1A), spent more time immobile (Fig.1B) and were less willing to self-care, compared with the mice in the control group (Fig.1C). Thus, DSS treatment induced an abnormal stress response in the IBD mice. Following intranasal OT treatment, the IBD mice recovered their motivation to explore, spent less time being immobile, and were more willing to self-care. However, the effects of intranasal OT were nullified in the intranasal OT + intranasal atosiban co-treated IBD mice (Fig.1A-C). 


\section{Intranasal OT restored DSS-induced hippocampal change of gene expression}

We next investigated the role of the nNOS/NO, BDNF and ERK signaling pathways of the hippocampus in abnormal stress-related behavior. We also examined whether intranasal OT attenuated DSS-induced abnormal stress-related behavior through these pathways ${ }^{2829,30}$. While the expression of nNOS/NO, OTR, $\mathrm{pERK} / \mathrm{ERK}$ and BDNF increased in the hippocampus of the IBD mice, intranasal OT treatment caused the expression of these genes to return to normal levels (Fig.2A-F). Again, intranasal atosiban co-treatment attenuated many of the effects of intranasal OT except its effect on OTR and BDNF expression (Fig.2C and F).

\section{Intranasal OT ameliorated DSS-induced intestinal inflammation}

In addition to improving abnormal stress-related behavior in the IBD mice, intranasal OT treatment ameliorated intestinal inflammation. Furthermore, the intranasal OT treated mice recovered their weight loss compared to the untreated IBD mice. Besides the intranasal OT treated mice showed an increase in colon length, decreased disease activity score, decreased histological score, decreased colon macroscopic damage score, and a reduction in the levels of TNF- $\alpha$ and IL-1 $\beta$ (Fig.3A-H). However, the intranasal OT + intranasal atosiban co-treated mice showed effects opposite to those observed in the intranasal OT mice (Fig.3A-H).

\section{Intranasal OT reverted the changes induced by DSS in HPA axis and the sympathetic-adrenal medulla (SAM) axis}

Communication between the gut and the brain is largely dependents on different pathways: the HPA axis; neural communication (vagal and sympathetic system); and systemic communication which include peptides (such as OT) and other small molecules ${ }^{31}$. In the next stage of our study, we investigated the activity of these different pathways. For HPA axis pathway, the enzyme-linked immunosorbent assay (ELISA) analysis demonstrated that the levels of corticotropin-releasing hormone $(\mathrm{CRH})$ were up-regulated in the hypothalamus of the IBD mice in response to DSS-induced inflammation response compared to those in the control mice and that intranasal OT treatment decreased the expression of CRH. Similar results were obtained with adreno-cortico-tropic-hormone (ACTH) and cortisol(Fig.4A-C). Again, these changes were diminished by intranasal atosiban co-treatment(Fig.4A-C). For the systemic communication pathway, we detected the expression of OT in the hypothalamus and OT levels in serum. Endogenous OT is known to play a role in intestinal immunity, and intraperitoneal injection of OT can repair intestinal injury ${ }^{32}$, While the OT levels in serum increased in the IBD mice, the expression of OT in the hypothalamus did not change (Fig.4D-E). Interestingly, although intranasal OT increased expression of OT in the hypothalamus, the OT levels in serum did not increase following intranasal OT treatment (Fig.4D-E). For the neural communication pathways, we analyzed the catecholamine levels in serum using ELISA to assess the activity of the sympathetic system. The results showed that the catecholamine levels were elevated in IBD mice, and that, in comparison, intranasal OT suppressed catecholamine levels in serum in comparison (Fig.4F). In the vagus nerve system, we decided to focus on the vago-splenic pathway, and in particular, the cholinergic antiinflammation pathway $(\mathrm{CAP})^{33}$. Compared with the control group, DSS-induced colitis was associated with a decrease in acetylcholine(ACh)and an increase of TNF- $\alpha$ in the spleen (Fig.4G-H). Intranasal OT treatment reversed the observed decrease in $\mathrm{ACh}$ and increase in $\mathrm{TNF}-\alpha$ in the spleen of mice with DSS-induced colitis (Fig.4G-H).

\section{Intranasal OT attenuated the DSS-induced changes in macrophage cell, NK cell and Treg cell populations in the spleen}

To investigate the mechanism underlying the effects of intranasal OT on IBD mice, we performed a flow cytometry analysis of M1 and M2 type macrophages, NKp46+NK cells and Treg cells isolated from spleens obtained from mice in the different treatment groups. DSS treatment remarkably augmented the percentages of the M1 and M2 type macrophages in the spleen, while it simultaneously decreased the percentage of Treg cell (Fig.5A, B and D). Intranasal OT administration further increased the percentages of M1 and M2 type macrophages in the spleen, increased the percentage of Treg cells and decreased the M1/M2 ratio and the percentage of NKp46+NK cell (Fig.5A-D). 


\section{Splenectomy abolished the protective effects of intranasal OT against DSS-induced abnormal stress-related behavior and intestinal inflammation}

Stress-related behavior and colitis severity were similar between the DSS-treated mice who had undergone splenectomy and the non-operated animals. However, the protective effects of intranasal OT administration, in particular, its effect on mobilization time, immobilization time, weight change, colon length, disease activity score, histological score, colon macroscopic damage score, were negated (Fig.6A-I).

\section{Discussion}

Psychological disorders have long been related to gastrointestinal dysfunction. For example, patients with IBD have more opportunity to suffer from anxiety and depression, in contrast, children with autism spectrum disorders have a higher prevalence of $\mathrm{CD}$ and $\mathrm{UC}^{7,34,35}$. Evidence have now been published to support the hypothesis that intestinal disorders can lead to psychological disorders, including depression and anxiety, and vice versa. This mutual effect is mediated by bidirectional communications involving reciprocal signaling between the brain and gut. In our experiments, DSS-induced colitis invoked an abnormal stress response in mice. Compared with mice in the control group, the IBD mice were immobile for long periods and were less motivated to perform self-care activities. While immobilization reflects a reduced willingness to explore, mobilization and grooming reflect motivation and beneficial self-care. The cause of stress-related behavior in mice with colitis appears to be the activation of nNOS/ NO pathway. In a previous report, an elevation in the nNOS/NO activity in the hippocampus was shown to account for stress-induced anxiety behavior ${ }^{28}$. As a consequence of the increased nNOS/ NO activity, increase in OTR, ERK and BDNF expression may be expected in order to alleviate the stress response. In addition, previous research has demonstrated that BDNF mRNA and protein expression in the hippocampus increased after chronic restraint stress ${ }^{29}$. The increased NO level leads to neurodegeneration, the induction of acidosis, and inflammation. Morris et al . reported that the increased NO impaired mitochondrial function, contributing to depression ${ }^{36}$. In the central nervous system, nNOS neurons co-exist with OT in the hypothalamus ${ }^{37}$. Therefore, a decrease in the NO level after intranasal OT administration may facilitate recovery from intestinal inflammation. In addition to the above mechanisms, other brain regions, and other genes, may be involved in the behavioral changes observed after intranasal OT administration. However, the importance of additional mechanisms has not been explored.

In the present study, we confirmed that DSS-induced colitis could activate the HPA axis. Moreover, we show that subsequent intranasal OT administration can inhibit this HPA axis activation. Considering the anti-inflammatory properties of glucocorticoids, the observed elevation in plasma cortisol levels is likely a manifestation of the restoration of homeostasis. However, superabundant cortisol levels have been shown to increase vulnerability to psychological disorders ${ }^{38}$. Indeed, hypercortisolism is a common finding in patients with depression and anxiety ${ }^{3940}$. Thus, the inflammatory response caused an increase of cortisol. Elevated cortisol crossed the blood-brain barrier to enter the brain, which leads to increase of negative factor (nNOS/NO) and compensatory protective factors (BDNF). Intranasal OT administration also increased the concentration of OT level in the hypothalamus. This externally administered OT may activate the hypothalamus neurons to release more OT through an autofeedback mechanism ${ }^{41}$. OT can also prevent transcription of $\mathrm{CRH}$ in the hypothalamus by interfering with promotor activity ${ }^{42}$. Thus, changes in the HPA axis and alleviation of intestinal inflammation may be attributed to intranasal OT. Increased serum OT caused by DSS-induced colitis may be a compensatory response of the body considering the protective role of OT in intestinal inflammation. Importantly, our results showed that increased OT expression in the hypothalamus did not result in increased OT levels in the periphery. Since we can also exclude the possibility that intranasally administered OT was released into the blood, OT must exert its influences through another pathway.

The induction and development of IBD disturbs the balance between the sympathetic and vagal nervous systems which are responsible for the maintenance of homeostasis ${ }^{43,44}$. In IBD patients, this autonomic dysfunction manifests itself in higher concentrations of serum catecholamine and low heart rate variability ${ }^{4,45,46}$. As a component of the sympathetic nervous system, catecholamine plays a crucial role in maintaining homeosta- 
sis in the gut ${ }^{47}$. Our results show a fluctuation in catecholamine levels consistent with the changes in HPA activity. We hypothesize that that intranasal OT activates neurons in the dorsal vagal complex (DVC), the primary center of the CAP that regulates gastrointestinal immunity. The DVC is known to express OTR and microinjection of OT into the DVC can enhance gut motility ${ }^{48-50}$. In future studies, we aim to investigate whether intranasal OT can enhance the activity of DVC and if so, to determine the underlying mechanism.

An elevation in the M1 and M2 macrophages in the spleen of the IBD mice and a decrease in the M1/M2 ratio have both been reported in earlier research ${ }^{51}$. The M2 macrophages, which possess anti-inflammatory activity, are known to reside in the gut and to counteract intestinal inflammation ${ }^{52}$. However, not much is known about the macrophage activity in lymphoid organs outside the gut (e.g. spleen). For the first time, we reported here that intranasal OT administration could shift the M1/M2 equilibrium in the spleen of DSStreated mice toward the M2 type. The classic mechanism in the vago-splenic pathway involved the release of ACh from T lymphocytes residing in the spleen, Ach activation $\alpha 7 \mathrm{nAChR}$ channels on macrophages, and inhibition of release of TNF- $\alpha^{53}$. In the gut, ACh promotes macrophage polarization to the M2 phenotype in the gut ${ }^{54,55}$. The observed decrease in TNF- $\alpha$ level and increase in Ach level in the spleen after intranasal OT administration are consistent with the intranasal OT activation of the vago-splenic pathway (part of the CAP). NKp46(+) NK cells have been reported to be increased in the intestinal mucosa of patients with CD compared with controls ${ }^{56}$. However, the role of splenic NKp46+NK cells in the regulation of gut inflammation is unknown. For the first time, our results indicate that DSS-induced colitis did not change the expression of peripheral NKp46+NK cells. Moreover, we specifically demonstrated that intranasal OT administration down-regulated NKP46+NK cells in the spleen. We speculate that the decrease in NKp46+NK cells in the spleen was related to activation of the vago-splenic pathway. DSS-induced colitis did reduce the percentage of Treg cells in the spleen. Similarly, patients with IBD exhibited reduced numbers of peripheral Treg cells ${ }^{57}$. Peripheral Treg cells have been reported to serve as a protective mechanism in IBD $^{58}$. In a model of viral myocarditis, activation of the cholinergic anti-inflammatory pathway increased the portion of Treg cell in the spleen ${ }^{59}$. The observed increase in Treg cells in DSS-treated mice after intranasal OT administration may be related to the decrease of TNF- $\alpha$ and increase of $\mathrm{ACh}^{2760}$.

Splenectomy did not affect DSS-induced colitis, which means colitis is independent from spleen involvement. This observation was in accord with the hypothesis that intestinal immune tissue, but not the spleen, contributes to the development of colitis $^{61}$. In the DSS-treated mice, who had undergone a splenectomy, attenuation of colitis severity or of abnormal behaviors was not observed after intranasal OT administration. Furthermore, while inflammation is initiated from the gut, regulation of circulating immune cells and the release of cytokine are mediated by the spleen. The beneficial effects of intranasal OT administration on DSS-induced colitis mice were lost following splenectomy in our mice model. Thus, intranasal OT required spleen mediation. Elimination of cholinergic anti-inflammatory efficacy in mice following splenectomy has previously been demonstrated in both DSS- and DNBS- induced intestinal inflammation, lethal endotoxemia, polymicrobial sepsis and kidney ischemia-reperfusion injury ${ }^{62636465}$. It should also be acknowledged that the vagus nerve innervates the proximal colon in addition to the spleen. Therefore, it is theoretically possible that intranasal OT administration altered enteric nervous system activity in the remainder of the colon ${ }^{66}$, and that this, in turn, may result in alterations in immune cell activities, cytokines release, and suppression of inflammation. This could also account for the effects of vagus nerve signaling on colitis that were observed in this and other studies ${ }^{6768}$.

However, the speculation about colon participation should be proven. In future studies, we aim to investigate the effects of intranasal OT administration on the enteric nervous system.

Further, our study has several limitations. Firstly, a direct correlation between changes in OT and neuron activity in the DVC has not been shown. Moreover, we did not investigate the relationship between intranasal OT administration and the enteric nervous system. Secondly, the route, timing, dosage and side effects of intranasal OT treatment should be elucidated. Finally, our study only reports data from an IBD mouse model. Few studies have investigated intranasal OT administration in IBD patients. Thus, additional research in IBD patients is required. In conclusion, our study demonstrates that intranasal OT administration 
significantly attenuates DSS-induced abnormal stress-related behavior and intestinal inflammation. We conclude that intranasal OT administration may provide a basis for the treatment of both exaggerated stress responses and intestinal diseases in the future.

Abbreviations

IBD: inflammatory bowel disease

CD: Crohn's disease

UC: ulcerative colitis

HPA axis: hypothalamic-pituitary-adrenal axis

OT: oxytocin

OTR: oxytocin receptor

Treg: regulatory $\mathrm{T}$

DSS: dextran sodium sulfate

WAST: water avoidance stress test

SAM axis: sympathetic-adrenal medulla axis

$\mathrm{CRH}$ : corticotropin-releasing hormone

ACTH: adreno-cortico-tropic-hormone

ELISA: enzyme-linked immunosorbent assay

Ach: acetylcholine

CAP: cholinergic anti-inflammation pathway

DVC: dorsal vagal complex

\section{Reference}

1 Baumgart, D. C. \& Carding, S. R. Inflammatory bowel disease: cause and immunobiology. The Lancet369 , 1627-1640, doi:10.1016/s0140-6736(07)60750-8 (2007).

2 Graff, L. A. et al. Stress coping, distress, and health perceptions in inflammatory bowel disease and community controls. The American journal of gastroenterology104, 2959-2969, doi:10.1038/ajg.2009.529 (2009).

3 Schoultz, M., Atherton, I. \& Watson, A. Mindfulness-based cognitive therapy for inflammatory bowel disease patients: findings from an exploratory pilot randomised controlled trial. Trials $\mathbf{1 6}$, 379, doi:10.1186/s13063-015-0909-5 (2015).

4 Neuendorf, R., Harding, A., Stello, N., Hanes, D. \& Wahbeh, H. Depression and anxiety in patients with Inflammatory Bowel Disease: A systematic review. Journal of psychosomatic research 87 , 70-80, doi:10.1016/j.jpsychores.2016.06.001 (2016).

5 Kim, M. C. et al. Factors Associated with Anxiety and Depression in Korean Patients with Inactive Inflammatory Bowel Disease. Gut and liver 10 , 399-405, doi:10.5009/gnl15188 (2016).

6 Ananthakrishnan, A. N. et al.Association between depressive symptoms and incidence of Crohn's disease and ulcerative colitis: results from the Nurses' Health Study.Clinical gastroenterology and hepatology : the official clinical practice journal of the American Gastroenterological Association11 , 57-62, doi:10.1016/j.cgh.2012.08.032 (2013). 
7 Gracie, D. J., Hamlin, P. J. \& Ford, A. C. Longitudinal impact of IBS-type symptoms on disease activity, healthcare utilization, psychological health, and quality of life in inflammatory bowel disease. The American journal of gastroenterology, doi:10.1038/s41395-018-0021-z (2018).

8 Mikocka-Walus, A., Pittet, V., Rossel, J. B. \& von Kanel, R. Symptoms of Depression and Anxiety Are Independently Associated With Clinical Recurrence of Inflammatory Bowel Disease. Clinical gastroenterology and hepatology : the official clinical practice journal of the American Gastroenterological Association 14 , 829-835.e821, doi:10.1016/j.cgh.2015.12.045 (2016).

9 Gracie, D. J., Guthrie, E. A., Hamlin, P. J. \& Ford, A. C. Bi-directionality of Brain-Gut Interactions in Patients With Inflammatory Bowel Disease. Gastroenterology154 , 1635-1646 e1633, doi:10.1053/j.gastro.2018.01.027 (2018).

10 Gracie, D. J. et al. Effect of psychological therapy on disease activity, psychological comorbidity, and quality of life in inflammatory bowel disease: a systematic review and meta-analysis. The lancet. Gastroenterology $E_{3}$ hepatology 2 , 189-199, doi:10.1016/S2468-1253(16)30206-0 (2017).

11 Husain, A. \& Triadafilopoulos, G. Communicating with patients with inflammatory bowel disease.Inflammatory bowel diseases 10, 444-450; discussion 451 (2004).

12 Brownstein, M. J., Russell, J. T. \& Gainer, H. Synthesis, transport, and release of posterior pituitary hormones. Science 207, 373-378 (1980).

13 Pohl, T. T., Young, L. J. \& Bosch, O. J. Lost connections: Oxytocin and the neural, physiological, and behavioral consequences of disrupted relationships.International journal of psychophysiology : official journal of the International Organization of Psychophysiology, doi:10.1016/j.ijpsycho.2017.12.011 (2018).

14 Augustine, R. A., Seymour, A. J., Campbell, R. E., Grattan, D. R. \& Brown, C. H. Integrative neurohumoral regulation of oxytocin neuron activity in pregnancy and lactation. Journal of neuroendocrinology , doi:10.1111/jne.12569 (2018).

15 Carter, C. S. The Oxytocin-Vasopressin Pathway in the Context of Love and Fear.Frontiers in endocrinology 8,356, doi:10.3389/fendo.2017.00356 (2017).

16 Misrani, A., Tabassum, S. \& Long, C. Oxytocin system in neuropsychiatric disorders: Old concept, new insights. Sheng li xue bao : [Acta physiologica Sinica]69 , 196-206 (2017).

$17 \mathrm{Li}, \mathrm{Q}$. et al. Oxytocin Exerts Antidepressant-like effect by potentiating dopaminergic synaptic transmission in the mPFC. Neuropharmacology 162, 107836, doi:10.1016/j.neuropharm.2019.107836 (2020).

18 Banks, W. A. Peptides and the blood-brain barrier. Peptides $\mathbf{7 2}$, 16-19, doi:10.1016/j.peptides.2015.03.010 (2015).

19 Ermisch, A., Ruhle, H. J., Landgraf, R. \& Hess, J. Blood-brain barrier and peptides. Journal of cerebral blood flow and metabolism : official journal of the International Society of Cerebral Blood Flow and Metabolism 5 , 350-357, doi:10.1038/jcbfm.1985.49 (1985).

20 Leng, G. \& Ludwig, M. Intranasal Oxytocin: Myths and Delusions. Biological psychiatry 79 , 243-250, doi:10.1016/j.biopsych.2015.05.003 (2016).

21 Lee, M. R. et al. Labeled oxytocin administered via the intranasal route reaches the brain in rhesus macaques. Nature communications 11, 2783, doi:10.1038/s41467-020-15942-1 (2020).

22 Anagnostou, E. et al.Intranasal oxytocin in the treatment of autism spectrum disorders: a review of literature and early safety and efficacy data in youth.Brain research 1580 , 188-198, doi:10.1016/j.brainres.2014.01.049 (2014).

23 Reichmann, F. et al.Dextran sulfate sodium-induced colitis alters stress-associated behaviour and neuropeptide gene expression in the amygdala-hippocampus network of mice. Scientific reports $\mathbf{5}$, 9970, 
doi:10.1038/srep09970 (2015).

24 Sanu, O. \& Lamont, R. F. Critical appraisal and clinical utility of atosiban in the management of preterm labor. Therapeutics and clinical risk management 6 , 191-199, doi:10.2147/tcrm.s9378 (2010).

25 Yamasue, H. et al. Effect of intranasal oxytocin on the core social symptoms of autism spectrum disorder: a randomized clinical trial. Molecular psychiatry, doi:10.1038/s41380-018-0097-2 (2018).

26 Liu, R., Yuan, X., Chen, K., Jiang, Y. \& Zhou, W. Perception of social interaction compresses subjective duration in an oxytocin-dependent manner. eLife 7 , doi:10.7554/eLife.32100 (2018).

27 Grandi, A. et al. alpha7 Nicotinic Agonist AR-R17779 Protects Mice against 2,4,6-Trinitrobenzene Sulfonic Acid-Induced Colitis in a Spleen-Dependent Way. Frontiers in pharmacology 8 , 809, doi:10.3389/fphar.2017.00809 (2017).

28 Zhu, L. J. et al.Hippocampal nuclear factor kappa B accounts for stress-induced anxiety behaviors via enhancing neuronal nitric oxide synthase (nNOS)-carboxy-terminal PDZ ligand of nNOS-Dexras1 coupling. Journal of neurochemistry 146 , 598-612, doi:10.1111/jnc.14478 (2018).

29 Bennett, M. R. \& Lagopoulos, J. Stress and trauma: BDNF control of dendritic-spine formation and regression. Progress in neurobiology 112, 80-99, doi:10.1016/j.pneurobio.2013.10.005 (2014).

30 Bali, A., Randhawa, P. K. \& Jaggi, A. S. Stress and opioids: role of opioids in modulating stress-related behavior and effect of stress on morphine conditioned place preference. Neuroscience and biobehavioral reviews51 , 138-150, doi:10.1016/j.neubiorev.2014.12.018 (2015).

31 Rogers, G. B. et al. From gut dysbiosis to altered brain function and mental illness: mechanisms and pathways. Molecular psychiatry 21, 738-748, doi:10.1038/mp.2016.50 (2016).

32 Chen, D. et al. Oxytocin evokes a pulsatile PGE2 release from ileum mucosa and is required for repair of intestinal epithelium after injury. Scientific reports5 , 11731, doi:10.1038/srep11731 (2015).

33 Bonaz, B., Sinniger, V. \& Pellissier, S. The Vagus Nerve in the Neuro-Immune Axis: Implications in the Pathology of the Gastrointestinal Tract. Frontiers in immunology 8 , 1452, doi:10.3389/fimmu.2017.01452 (2017).

34 Gracie, D. J., Guthrie, E. A., Hamlin, P. J. \& Ford, A. C. Bi-directionality of Brain-Gut Interactions in Patients With Inflammatory Bowel Diseases. Gastroenterology, doi:10.1053/j.gastro.2018.01.027 (2018).

35 Lee, M. et al. Association of Autism Spectrum Disorders and Inflammatory Bowel Disease. Journal of autism and developmental disorders, doi:10.1007/s10803-017-3409-5 (2017).

36 Morris, G. et al.Nitrosative Stress, Hypernitrosylation, and Autoimmune Responses to Nitrosylated Proteins: New Pathways in Neuroprogressive Disorders Including Depression and Chronic Fatigue Syndrome. Molecular neurobiology 54, 4271-4291, doi:10.1007/s12035-016-9975-2 (2017).

37 Nylen, A. et al. Nitric oxide synthase in the hypothalamic paraventricular nucleus of the female rat; organization of spinal projections and coexistence with oxytocin or vasopressin. Brain research 908, 10-24, doi:10.1016/s0006-8993(01)02539-2 (2001).

38 Labad, J. The role of cortisol and prolactin in the pathogenesis and clinical expression of psychotic disorders. Psychoneuroendocrinology 102, 24-36, doi:10.1016/j.psyneuen.2018.11.028 (2019).

39 Schatzberg, A. F. Anna-Monika Award Lecture, DGPPN Kongress, 2013: the role of the hypothalamicpituitary-adrenal (HPA) axis in the pathogenesis of psychotic major depression. The world journal of biological psychiatry : the official journal of the World Federation of Societies of Biological Psychiatry 16 , 2-11, doi:10.3109/15622975.2014.916414 (2015). 
40 Stetler, C. \& Miller, G. E. Depression and hypothalamic-pituitary-adrenal activation: a quantitative summary of four decades of research. Psychosomatic medicine 73 , 114-126, doi:10.1097/PSY.0b013e31820ad12b (2011).

41 Wang, Y. F., Ponzio, T. A. \& Hatton, G. I. Autofeedback effects of progressively rising oxytocin concentrations on supraoptic oxytocin neuronal activity in slices from lactating rats. American journal of physiology. Regulatory, integrative and comparative physiology 290, R1191-1198, doi:10.1152/ajpregu.00725.2005 (2006).

42 Jurek, B. et al. Oxytocin Regulates Stress-Induced Crf Gene Transcription through CREB-Regulated Transcription Coactivator 3. The Journal of neuroscience : the official journal of the Society for Neuroscience 35 , 12248-12260, doi:10.1523/JNEUROSCI.1345-14.2015 (2015).

43 Willemze, R. A., Luyer, M. D., Buurman, W. A. \& de Jonge, W. J. Neural reflex pathways in intestinal inflammation: hypotheses to viable therapy. Nature reviews. Gastroenterology $\& 3$ hepatology 12, 353-362, doi:10.1038/nrgastro.2015.56 (2015).

44 Ciesielczyk, K., Furgala, A., Dobrek, L., Juszczak, K. \& Thor, P. Altered sympathovagal balance and pain hypersensitivity in TNBS-induced colitis. Archives of medical science : AMS 13 , 246-255, doi:10.5114/aoms.2015.55147 (2017).

45 Martin, F. P. et al.Urinary metabolic insights into host-gut microbial interactions in healthy and IBD children. World journal of gastroenterology23 , 3643-3654, doi:10.3748/wjg.v23.i20.3643 (2017).

46 Sharma, P., Makharia, G. K., Ahuja, V., Dwivedi, S. N. \& Deepak, K. K. Autonomic dysfunctions in patients with inflammatory bowel disease in clinical remission.Digestive diseases and sciences 54, 853-861, doi:10.1007/s10620-008-0424-6 (2009).

47 Mittal, R. et al.Neurotransmitters: The Critical Modulators Regulating Gut-Brain Axis.Journal of cellular physiology 232, 2359-2372, doi:10.1002/jcp.25518 (2017).

48 Zhao, D. Q. \& Ai, H. B. Oxytocin and vasopressin involved in restraint water-immersion stress mediated by oxytocin receptor and vasopressin $1 \mathrm{~b}$ receptor in rat brain. PloS one 6 , e23362, doi:10.1371/journal.pone.0023362 (2011).

49 Ho, J. M. et al. Hindbrain oxytocin receptors contribute to the effects of circulating oxytocin on food intake in male rats. Endocrinology 155 , 2845-2857, doi:10.1210/en.2014-1148 (2014).

50 Liu, C. Y., Xie, D. P., Liu, K. J., Zhou, Y. Q. \& Liu, J. Z. Oxytocin microinjected into dorsal motor nucleus of the vagus excites gallbladder motility via NMDA receptor-NO-cGMP pathway. Brain research 1032 , 116-122, doi:10.1016/j.brainres.2004.10.057 (2005).

51 Zhang, H. et al.Helicobacter pylori Colonization Protects Against Chronic Experimental Colitis by Regulating Th17/Treg Balance. Inflammatory bowel diseases 24, 1481-1492, doi:10.1093/ibd/izy107 (2018).

$52 \mathrm{Na}$, Y. R., Stakenborg, M., Seok, S. H. \& Matteoli, G. Macrophages in intestinal inflammation and resolution: a potential therapeutic target in IBD. Nature reviews. Gastroenterology 83 hepatology $\mathbf{1 6}, 531-$ 543, doi:10.1038/s41575-019-0172-4 (2019).

53 Rosas-Ballina, M. et al.Acetylcholine-synthesizing T cells relay neural signals in a vagus nerve circuit. Science 334, 98-101, doi:10.1126/science.1209985 (2011).

54 Zhang, Q., Lu, Y., Bian, H., Guo, L. \& Zhu, H. Activation of the alpha7 nicotinic receptor promotes lipopolysaccharide-induced conversion of M1 microglia to M2. American journal of translational research $\mathbf{9}$, 971-985 (2017).

55 Ortiz, T. et al.Polyphenolic Maqui Extract as a Potential Nutraceutical to Treat TNBS-Induced Crohn's Disease by the Regulation of Antioxidant and Anti-Inflammatory Pathways. Nutrients 12 , doi:10.3390/nu12061752 (2020). 
56 Takayama, T. et al.Imbalance of NKp44(+)NKp46(-) and NKp44(-)NKp46(+) natural killer cells in the intestinal mucosa of patients with Crohn's disease.Gastroenterology 139 , 882-892, 892 e881-883, doi:10.1053/j.gastro.2010.05.040 (2010).

57 Eastaff-Leung, N., Mabarrack, N., Barbour, A., Cummins, A. \& Barry, S. Foxp3+ regulatory T cells, Th17 effector cells, and cytokine environment in inflammatory bowel disease.Journal of clinical immunology 30 , 80-89, doi:10.1007/s10875-009-9345-1 (2010).

58 Yamada, A. et al. Role of regulatory $\mathrm{T}$ cell in the pathogenesis of inflammatory bowel disease. World journal of gastroenterology 22, 2195-2205, doi:10.3748/wjg.v22.i7.2195 (2016).

$59 \mathrm{De}-\mathrm{Pu}, \mathrm{Z}$. et al. The cholinergic anti-inflammatory pathway ameliorates acute viral myocarditis in mice by regulating CD4(+) T cell differentiation.Virulence $\mathbf{9}$, 1364-1376, doi:10.1080/21505594.2018.1482179 (2018).

60 Furiati, S. C. et al. Th1, Th17, and Treg Responses are Differently Modulated by TNF-alpha Inhibitors and Methotrexate in Psoriasis Patients. Scientific reports 9, 7526, doi:10.1038/s41598-019-43899-9 (2019).

61 Krieglstein, C. F. et al.Role of appendix and spleen in experimental colitis. The Journal of surgical research 101 , 166-175, doi:10.1006/jsre.2001.6223 (2001).

$62 \mathrm{Ji}, \mathrm{H}$. et al. Central cholinergic activation of a vagus nerve-to-spleen circuit alleviates experimental colitis. Mucosal immunology 7 , 335-347, doi:10.1038/mi.2013.52 (2014).

63 Huston, J. M. et al.Splenectomy inactivates the cholinergic antiinflammatory pathway during lethal endotoxemia and polymicrobial sepsis. The Journal of experimental medicine 203 , 1623-1628, doi:10.1084/jem.20052362 (2006).

64 Inoue, T. et al. Vagus nerve stimulation mediates protection from kidney ischemia-reperfusion injury through alpha7nAChR + splenocytes. The Journal of clinical investigation 126 , 1939-1952, doi:10.1172/JCI83658 (2016).

$65 \mathrm{Ma}$, J. et al.Dexmedetomidine-Mediated Prevention of Renal Ischemia-Reperfusion Injury Depends in Part on Cholinergic Anti-Inflammatory Mechanisms.Anesthesia and analgesia 130 , 1054-1062, doi:10.1213/ANE.0000000000003820 (2020).

66 Furness, J. B., Callaghan, B. P., Rivera, L. R. \& Cho, H. J. The enteric nervous system and gastrointestinal innervation: integrated local and central control.Advances in experimental medicine and biology 817 , 39-71, doi:10.1007/978-1-4939-0897-4_3 (2014).

67 Ghia, J. E., Blennerhassett, P., Kumar-Ondiveeran, H., Verdu, E. F. \& Collins, S. M. The vagus nerve: a tonic inhibitory influence associated with inflammatory bowel disease in a murine model. Gastroenterology 131 , 1122-1130, doi:10.1053/j.gastro.2006.08.016 (2006).

68 Hoover, D. B. Cholinergic modulation of the immune system presents new approaches for treating inflammation. Pharmacology \&3 therapeutics 179 , 1-16, doi:10.1016/j.pharmthera.2017.05.002 (2017).

\section{Figure legend}

Fig 1. IN OT improved the DSS-induced abnormal stress-related behavior of mice. During water avoidance stress exposure, DSS-induced IBD mice treated with intranasal oxytocin for 7 days showed (A) more activity, (B) less immobility and (C) more grooming behavior than untreated mice. IN atosiban reversed IN OT effects in IBD mice $(\mathrm{A}-\mathrm{C})$. Data are presented as means \pm SEM. one-way ANOVA, $\mathrm{n}=$ 6. ${ }^{* * *} \mathrm{P}<0.001$ for main effect: DSS versus normal control; \#\#\#P $<0.001$ for main effect: IN OT versus without IN OT in DSS-induced IBD mice; $\$ \$ \$ P<0.001$ for main effect: IN atosiban versus without IN atosiban of DSS-induced IBD mice treated with IN OT.

Fig 2. Intranasal OT restored DSS-induced hippocampal change of gene expression. A. Representative Western blots of hippocampal nNOS, ERK, pERK and OTR.B. Statistical graph of pERK/ERK 
ratio in different groups. C. Statistical graph of OTR ratio in different groups. D. Statistical graph of nNOS ratio in different groups. E. Protein expression of NO in hippocampus between different groups. F. Protein expression of BDNF in hippocampus between different groups. Data are presented as means +SEM. one-way ANOVA, $\mathrm{n}=3-6$. ${ }^{* *} \mathrm{P}<0.01$ and ${ }^{* * *} \mathrm{P}<0.001$ for main effect: DSS versus normal control; $\# \mathrm{P}<0.05$, \#\# $<0.01$, \#\#\# $<0.001$ and \#\#\#\#P $<0.0001$ for main effect: IN OT versus without IN OT in DSS-induced IBD mice; $\$ \mathrm{P}<0.05$ and $\$ \$ \$ \mathrm{P}<0.001$ for main effect: IN atosiban versus without IN atosiban of DSS-induced IBD mice treated with IN OT.

Fig 3. IN OT ameliorated DSS-induced intestinal inflammation. weight(A), colon length (B), disease Activity Score (C), expression of TNF- $\alpha$ (D)and IL-1 $\beta$ (E), histological Score (F), colon macroscopic damage score $(\mathrm{G})$ was assessed in different groups. H. Representative image of HE staining in different groups. Data are presented as means \pm SEM. one-way ANOVA, $\mathrm{n}=6-10$. ${ }^{* *} \mathrm{P}<0.01$ and ${ }^{* * *} \mathrm{P}<0.001$ for main effect: DSS versus normal control; $\# \mathrm{P}<0.05$ and \#\#\#P $<0.001$ for main effect: IN OT versus without IN OT in DSS-induced IBD mice; $\$ \mathrm{P}<0.05, \$ \$ \mathrm{P}<0.01$ and $\$ \$ \$ \mathrm{P}<0.001$ for main effect: IN atosiban versus without IN atosiban of DSS-induced IBD mice treated with IN OT.

Fig 4. Intranasal OT reverted the changes induced by DSS in HPA axis and the sympatheticadrenal medulla (SAM) axis. $\mathrm{CRH}$ in hypothalamus(A), $\mathrm{ACTH}(\mathrm{B})$, Cortisol(C), Catecholamine in serum(D), Oxytocin in hypothalamus(E), Oxytocin in $\operatorname{serum}(\mathrm{F}), \mathrm{Ach}(\mathrm{G})$ and $\mathrm{TNF}-\alpha(\mathrm{H})$ in spleen were assessed in different groups. Data are presented as means \pm SEM. one-way ANOVA, $\mathrm{n}=4-7 .{ }^{*} \mathrm{P}<0.05,{ }^{* *} \mathrm{P}<0.01$ and ${ }^{* * *} \mathrm{P}<0.001$ for main effect: DSS versus normal control; $\# \mathrm{P}<0.05$, \#\# $\mathrm{P}<0.01$ and \#\#\# $\mathrm{P}<0.001$ for main effect: IN OT versus without IN OT in DSS-induced IBD mice; NS: Without statistically significant; $\$ \mathrm{P}<0.05, \$ \$ \mathrm{P}<0.01$ and $\$ \$ \$ \mathrm{P}<0.001$ for main effect: IN atosiban versus without IN atosiban of DSS-induced IBD mice treated with IN OT.

Fig 5. IN OT attenuated the DSS-induced changes in macrophage cell, NK cell, and Treg cell populations in the spleen. Percentages of M1 macrophage (A), M2 macrophage(B), CD3-NKp46+NK cell (C), CD4+ CD25+foxp3+Treg cell(D) in the spleen. Data are presented as means +- SEM. one-way ANOVA, $\mathrm{n}=4-6$. ${ }^{* *} \mathrm{P}<0.01$ and ${ }^{* * *} \mathrm{P}<0.001$ for main effect: $\mathrm{DSS}$ versus normal control; $\# \mathrm{P}<0.05$ and $\# \# \mathrm{P}<0.01$ for main effect: IN OT versus without IN OT of DSS-induced IBD mice; $\$ \mathrm{P}<0.05$ and $\$ \$ \mathrm{P}<0.01$ for main effect: IN atosiban versus without IN atosiban of DSS-induced IBD mice treated with IN OT.

Fig 6.Splenectomy abolished the protective effects of IN OT against DSS-induced abnormal stress-related behavior and intestinal inflammation. A-C. Protective effects of IN OT on DSS-induced abnormal stress-related behavior was abolished by splenectomy. weight(D), colon length (E), disease activity score $(\mathrm{F})$, histological score $(\mathrm{G})$, colon macroscopic damage score $(\mathrm{H})$ was assessed in different groups. I. Representative image of HE staining in different groups. Data are presented as means +- SEM. two-way ANOVA, $\mathrm{n}=5$. ${ }^{* *} \mathrm{P}<0.01$ and ${ }^{* * *} \mathrm{P}<0.001$ for main effect: DSS versus normal control; $\# \mathrm{P}<0.05$ and $\# \# \mathrm{P}<0.01$ for main effect: IN OT versus without IN OT of DSS-induced IBD mice; $\$ \mathrm{P}<0.05$ and $\$ \$ \mathrm{P}<0.01$ for main effect: IN atosiban versus without IN atosiban of DSS-induced IBD mice treated with IN OT.

Fig 7. Possible mechanisms for IN OT improved DSS-induced abnormal stress-related behavior and intestinal inflammation.Intestinal inflammation "communicated" with brain through systemic communication, HPA axis and neural communication (vagal and sympathetic system), which induced psychological disorders. IN OT administration improved DSS-induced abnormal stress-related behavior and intestinal inflammation through HPA axis and cholinergic anti-inflammation pathway. 

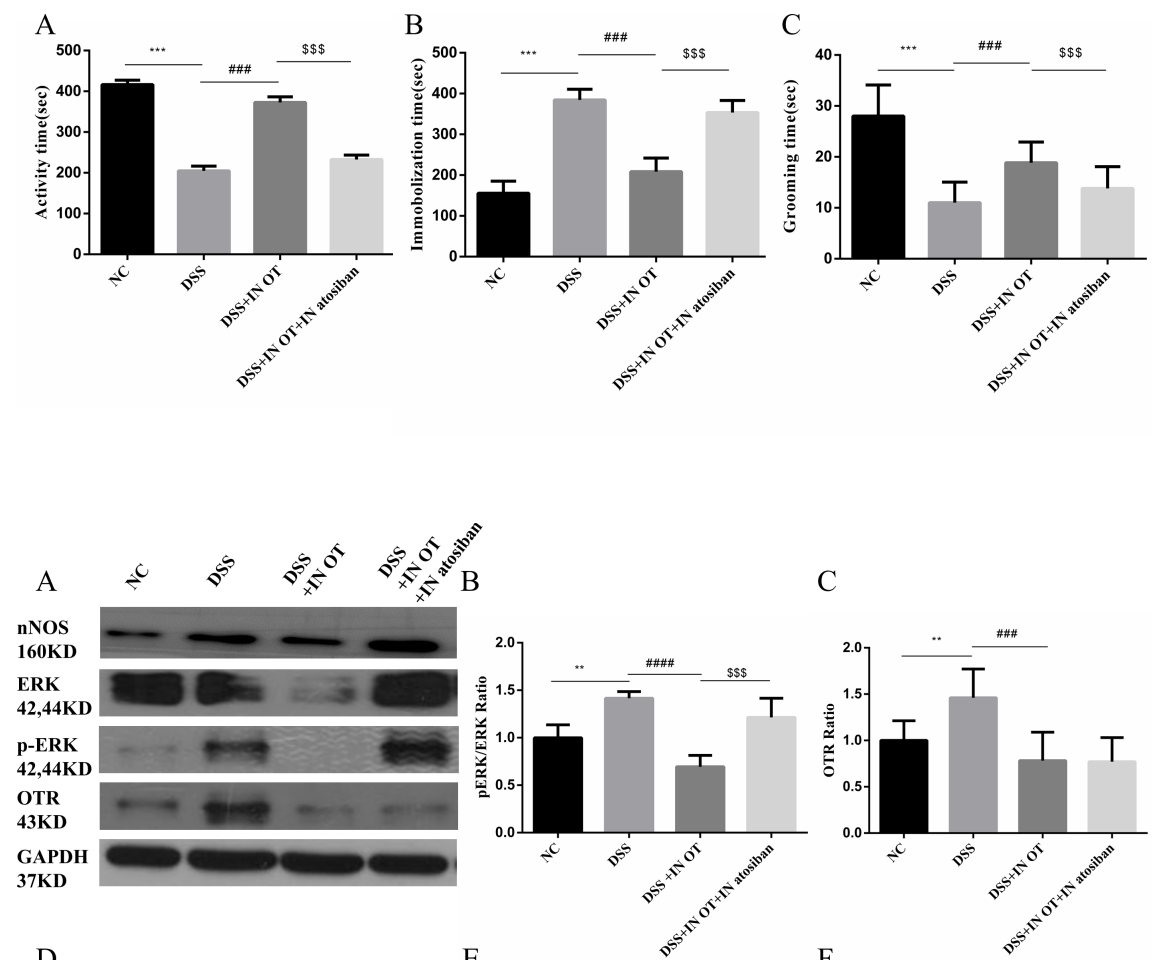

C

D

E
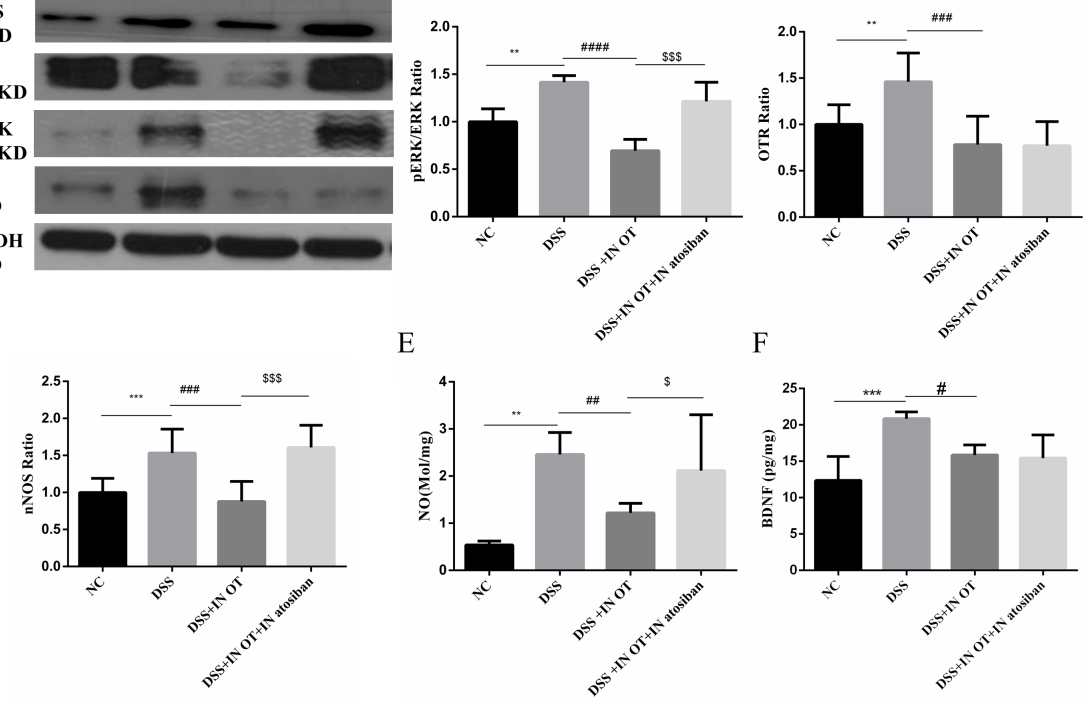


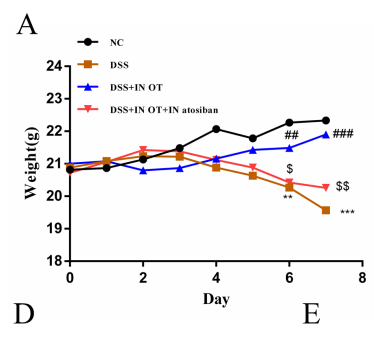

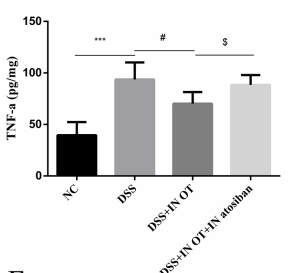

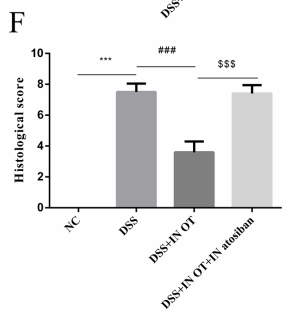

$\mathrm{G} \frac{\hat{\alpha}}{2}$
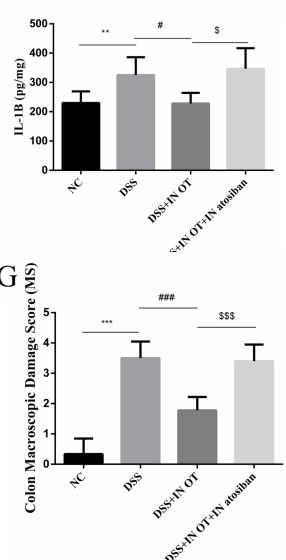

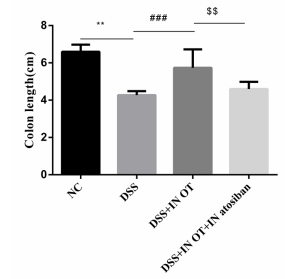

$\mathrm{H}$

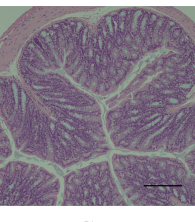

NC

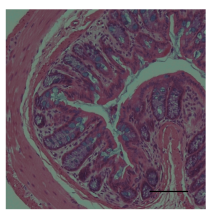

DSS+IN OT
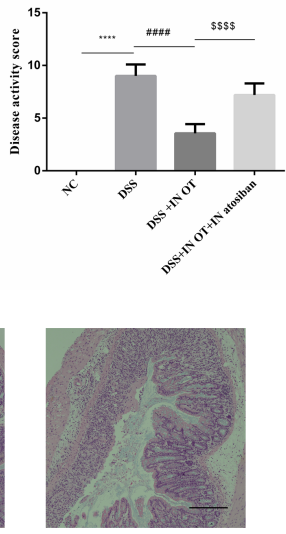

DSS

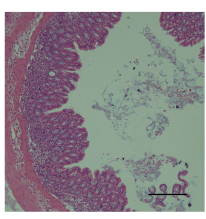

DSS+IN OT+IN atosiban

A

B

C

D

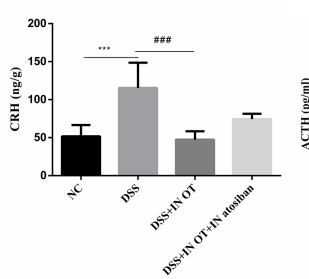

$\overbrace{0}^{T}$
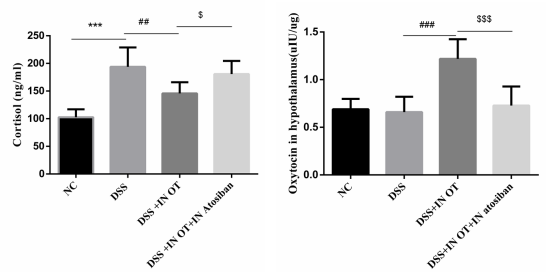

E

F

G

H

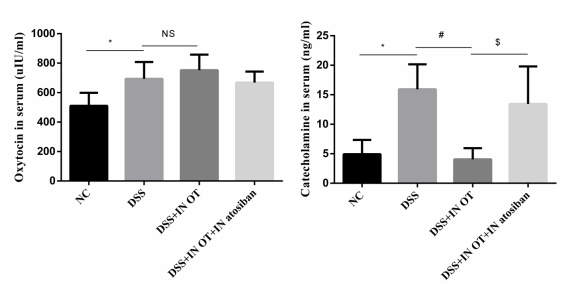

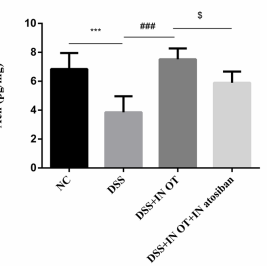

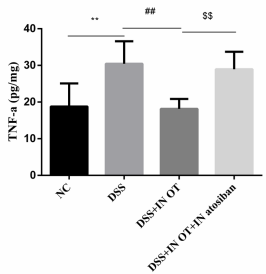



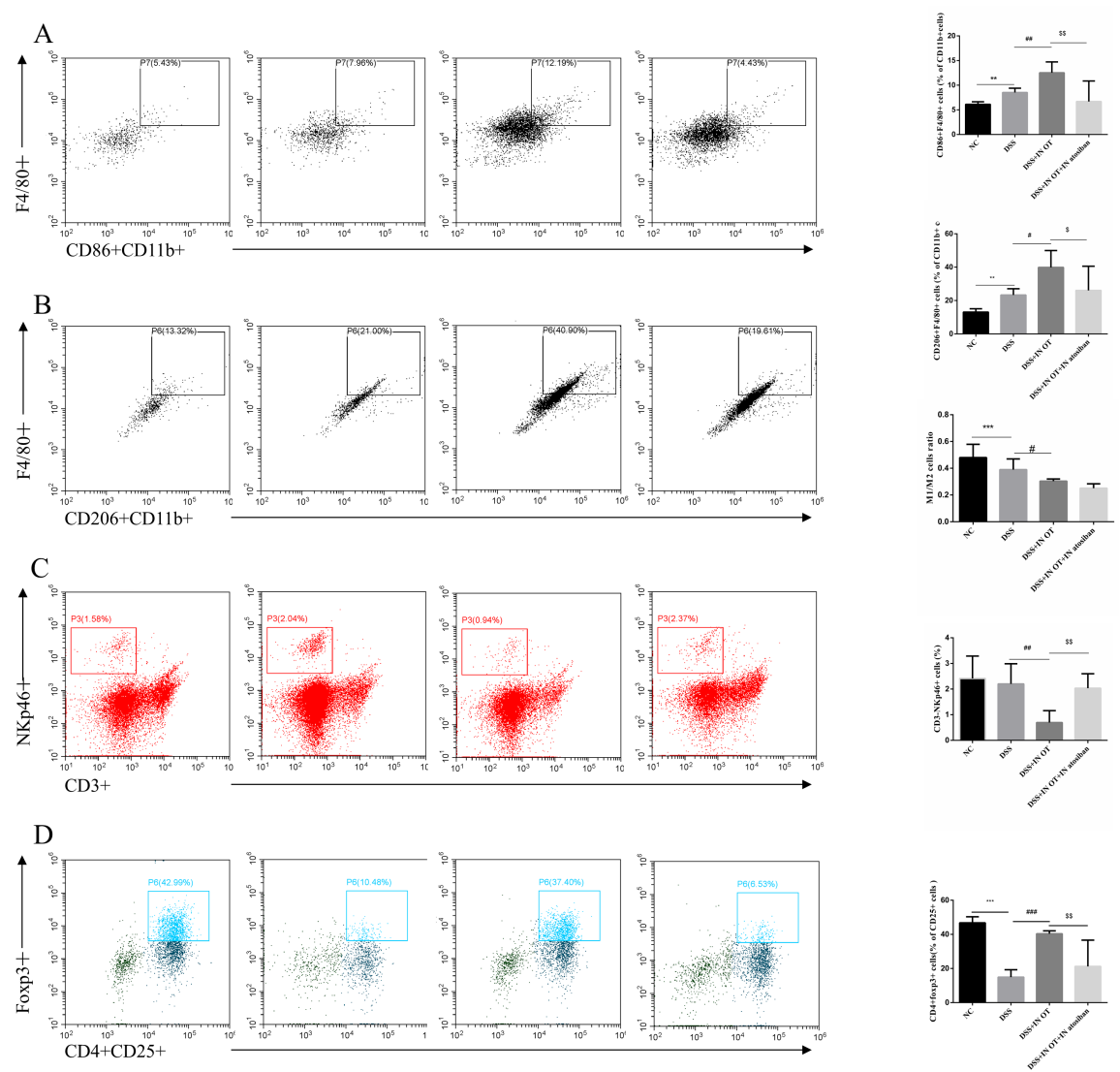
A

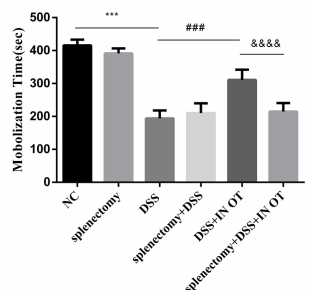

D

G

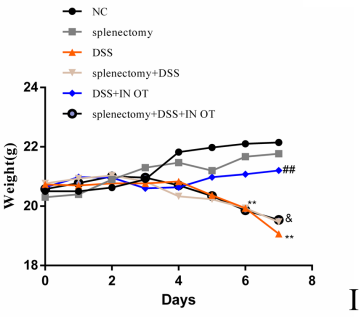

E
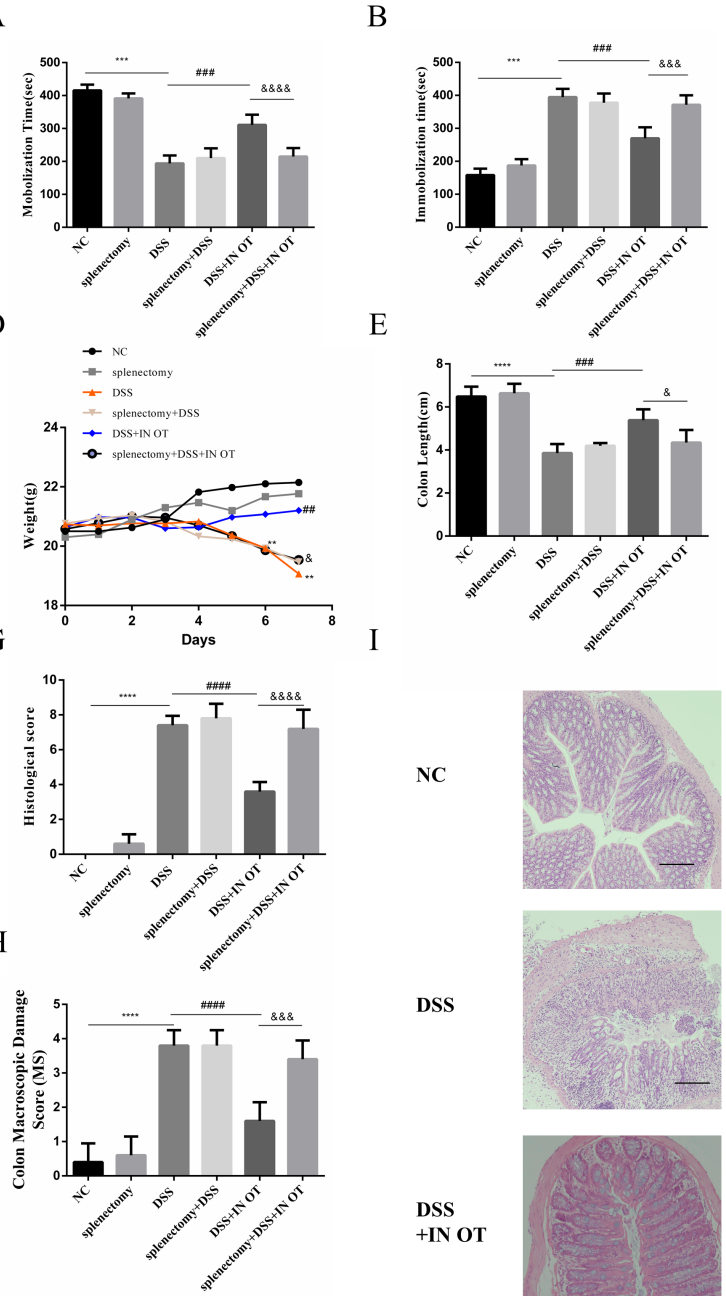

C

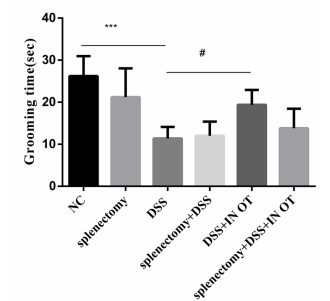

F

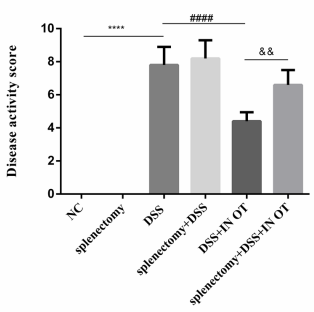

NC

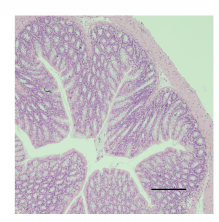

Splenectomy
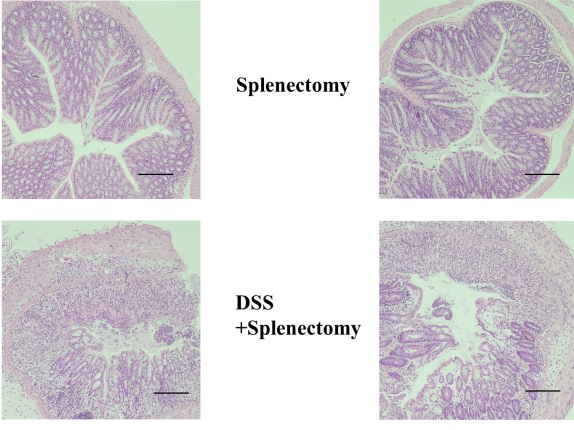

DSS

+ Splenectomy

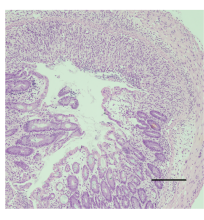

DSS
+ IN
o
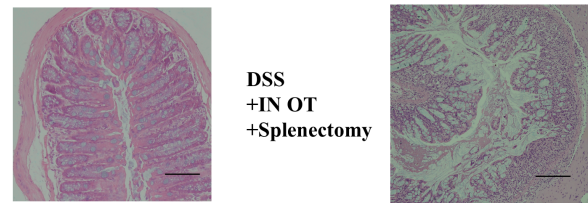


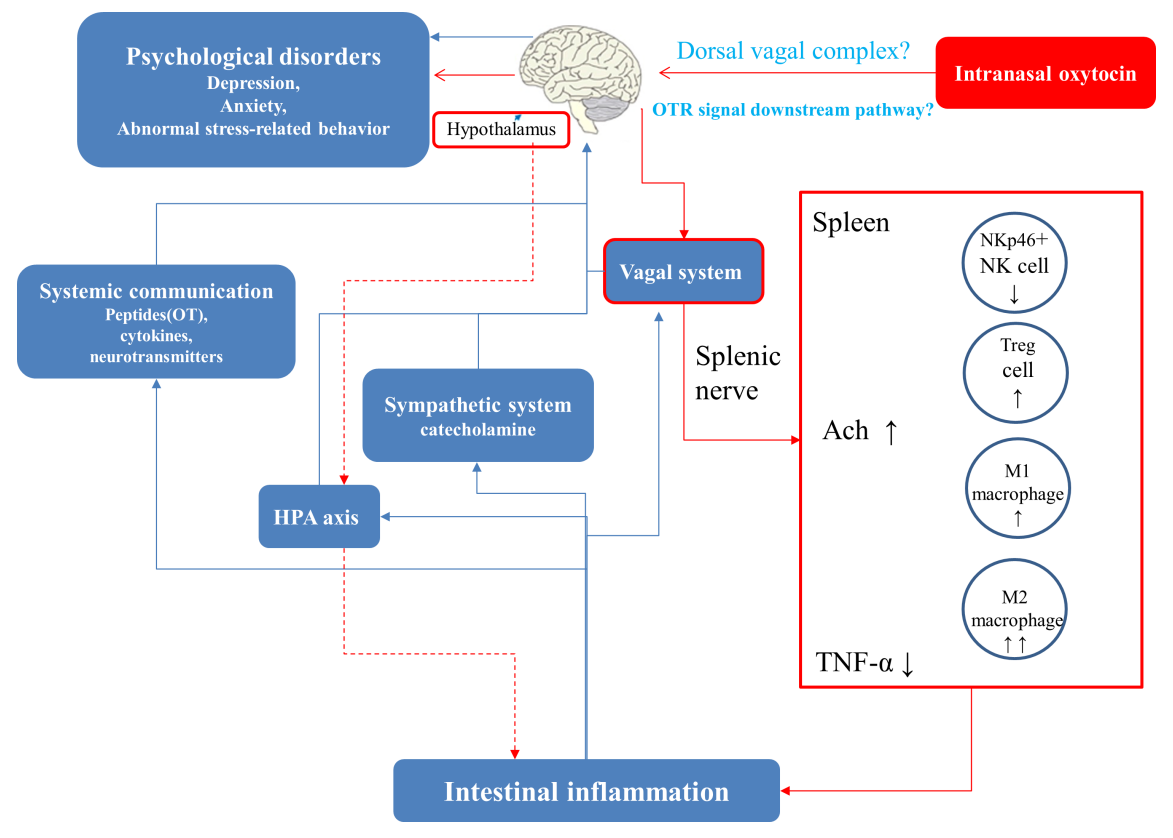




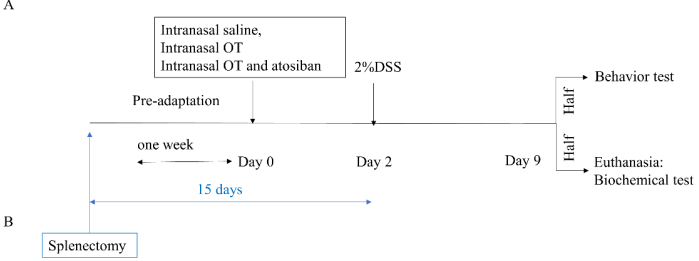

Supplementary Fig. Timeline and experiment design for behavior and biochemical tests. A. Mice were handled daily one week before the start of intranasal treatments to reduce stress associated with the procedure. 2 days before DSS-induced colitis, mice were received an intranasal saline, OT or OT with atosiban. At 7th day, half of each group were submitted to perform behavior test (water avoidance stress). The other half were sacrificed to perform biochemical test. B. Splenectomy was preformed 15 days before DSS-induced colitis. 